\title{
The Design of "Neutral" Carbanions with Intramolecular Charge Compensation
}

Chaitanya S. Wannere, Paul v. R. Schleyer, Henry F. Schaefer III*

Center for Computational Chemistry, University of Georgia, Athens, Georgia 30602.

Email: ccq@uga.edu

\section{Supporting Information:}

All the geometries provided below were optimized at B3LYP/6-311+G** using the Gaussian-03, Revision C.02, suite program.

Full author list for reference 16:

Frisch, M.J.; Trucks, G. W.; Schlegel, H. B.; Scuseria, G. E.; Robb, M. A.; Cheeseman, J. R.; Montgomery Jr., J. A.; Vreven, T.; Kudin, K. N.; Burant, J. C.; Millam, J. M.; Iyengar, S. S.; Tomasi, J.; Barone, V.; Mennucci, B.; Cossi, M.; Scalmani, G.; Rega, N.; Petersson, G. A.; Nakatsuji,H.; Hada, M.; Ehara, M.; Toyota, K.; Fukuda, R.; Hasegawa, J.; Ishida, M.; Nakajima, T.; Honda, Y.; Kitao, O.; Nakai, H.; Klene, M.; Li, X.; Knox, J. E.; Hratchian, H. P.; Cross, J. B.; Adamo, C.; Jaramillo,J.; Gomperts, R.; Stratmann, R. E.; Yazyev, O.; Austin, A. J.; Cammi, R.; Pomelli, C.; Ochterski, J. W.; Ayala, P. Y.; Morokuma, K.; Voth, G. A.; Salvador, P.; Dannenberg, J. J.; Zakrzewski, V. G.; Dapprich, S.; Daniels, A. D.; Strain, M. C.; Farkas, O.; Malick, D. K.; Rabuck, A. D.; Raghavachari, K.; Foresman, J. B.; Ortiz, J. V.; Cui, Q.; Baboul, A. G.; Clifford, S.; Cioslowski, J.; Stefanov, B. B.; Liu, G.; Liashenko, A.; Piskorz, P.; Komaromi, I.; Martin, R. L.; Fox, D. J.; Keith, T.; Al-Laham, M. A.; Peng, C. Y.; Nanayakkara, A.; Challacombe, M.; Gill, P. M.W.; Johnson, B.; Chen, W.; Wong,M. W.; Gonzalez, C.; Pople, J. A. Gaussian, Inc., Wallingford CT, 2004. 


Structure of Calicene:
$\begin{array}{llll}\mathrm{C} & -1.30704500 & 0.00000000 & 0.00000000 \\ \mathrm{C} & 0.04442200 & 0.00000000 & 0.00000000 \\ \mathrm{C} & -2.57015500 & 0.66193000 & -0.00000000 \\ \mathrm{C} & -2.57015500 & -0.66193000 & 0.00000000 \\ \mathrm{C} & 0.91140400 & 1.17047600 & -0.00000000 \\ \mathrm{C} & 0.91140400 & -1.17047600 & 0.00000000 \\ \mathrm{C} & 2.20283400 & 0.72705600 & -0.00000000 \\ \mathrm{C} & 2.20283400 & -0.72705600 & 0.00000000 \\ \mathrm{H} & -3.13679900 & 1.57871300 & -0.00000000 \\ \mathrm{H} & -3.13679900 & -1.57871300 & 0.00000000 \\ \mathrm{H} & 0.57076400 & 2.19653700 & -0.00000000 \\ \mathrm{H} & 0.57076400 & -2.19653700 & 0.00000000 \\ \mathrm{H} & 3.08940300 & 1.34715400 & -0.00000000 \\ \mathrm{H} & 3.08940300 & -1.34715400 & 0.00000000\end{array}$

\section{Structure of 1:}

$\begin{array}{lrrl}\mathrm{C} & 0.781091 & 1.232106 & 0.000000 \\ \mathrm{C} & 0.781091 & -1.232106 & 0.000000 \\ \mathrm{C} & 1.451444 & 0.000000 & 0.000000 \\ \mathrm{C} & -0.764338 & -1.187939 & 0.000000 \\ \mathrm{C} & -0.764338 & 1.187939 & 0.000000 \\ \mathrm{C} & -1.484950 & 0.000000 & 0.000000 \\ \mathrm{~N} & -1.297943 & -2.396573 & 0.000000 \\ \mathrm{~N} & -1.297943 & 2.396573 & 0.000000 \\ \mathrm{O} & 1.274463 & 2.371810 & 0.000000 \\ \mathrm{O} & 1.274463 & -2.371810 & 0.000000 \\ \mathrm{H} & 2.534616 & 0.000000 & 0.000000 \\ \mathrm{H} & -2.572352 & 0.000000 & 0.000000 \\ \mathrm{H} & -0.629379 & 3.164945 & 0.000000 \\ \mathrm{H} & -0.629379 & -3.164945 & 0.000000 \\ \mathrm{H} & -2.289655 & 2.571615 & 0.000000 \\ \mathrm{H} & -2.289655 & -2.571615 & 0.000000\end{array}$

\section{Structure of Bicalicene:}

$\begin{array}{lrrr}\mathrm{C} & 0.727821 & 1.861801 & 0.000000 \\ \mathrm{C} & -0.727821 & 1.861801 & 0.000000 \\ \mathrm{C} & 0.727821 & -1.861801 & 0.000000 \\ \mathrm{C} & -0.727821 & -1.861801 & 0.000000 \\ \mathrm{C} & 1.509206 & 0.711193 & 0.000000 \\ \mathrm{C} & -1.509206 & 0.711193 & 0.000000 \\ \mathrm{C} & 1.509206 & -0.711193 & 0.000000 \\ \mathrm{C} & -1.509206 & -0.711193 & 0.000000 \\ \mathrm{C} & 2.682396 & 0.000000 & 0.000000 \\ \mathrm{C} & -2.682396 & 0.000000 & 0.000000 \\ \mathrm{C} & -1.141664 & 3.211291 & 0.000000\end{array}$




$\begin{array}{lrrr}\mathrm{C} & 1.141664 & 3.211291 & 0.000000 \\ \mathrm{C} & -1.141664 & -3.211291 & 0.000000 \\ \mathrm{C} & 1.141664 & -3.211291 & 0.000000 \\ \mathrm{C} & 0.000000 & 4.027525 & 0.000000 \\ \mathrm{C} & 0.000000 & -4.027525 & 0.000000 \\ \mathrm{H} & -3.759356 & 0.000000 & 0.000000 \\ \mathrm{H} & 3.759356 & 0.000000 & 0.000000 \\ \mathrm{H} & -2.168134 & 3.551559 & 0.000000 \\ \mathrm{H} & 2.168134 & 3.551559 & 0.000000 \\ \mathrm{H} & -2.168134 & -3.551559 & 0.000000 \\ \mathrm{H} & 2.168134 & -3.551559 & 0.000000 \\ \mathrm{H} & 0.000000 & 5.108451 & 0.000000 \\ \mathrm{H} & 0.000000 & -5.108451 & 0.000000\end{array}$

\section{Structure of 2:}

\begin{tabular}{|c|c|c|c|}
\hline & 807 & 5868 & -2 \\
\hline 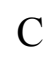 & -2.750845 & -1.632481 & -2.562703 \\
\hline $\mathrm{C}$ & -3.587225 & 1.082807 & -3.83114 \\
\hline $\mathrm{C}$ & -3.090936 & -1.362708 & -3.863934 \\
\hline C & -3.487904 & -0.133174 & -4.4349 \\
\hline & -2.824633 & 0.649414 & $-1.4398 \varepsilon$ \\
\hline 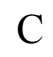 & -2.652607 & -0.777891 & -1.423415 \\
\hline $\mathrm{C}$ & 0.336438 & 1.162093 & 0.026603 \\
\hline $\mathrm{C}$ & 0.399185 & -1.151067 & -0.059786 \\
\hline $\mathrm{C}$ & 1.186433 & 0.000000 & 0.000000 \\
\hline $\mathrm{C}$ & -2.269945 & -1.415304 & -0.10925 \\
\hline C & -2.306335 & 1.359156 & -0.194723 \\
\hline $\mathrm{C}$ & -0.950826 & -0.723435 & 0.000000 \\
\hline & -0.971230 & 0.712408 & 0.033181 \\
\hline & 0.672656 & 2.190099 & 0.026634 \\
\hline H & 0.751043 & -2.173304 & -0.069371 \\
\hline $\mathrm{H}$ & 2.267590 & 0.021465 & 0.041167 \\
\hline H & -3.749325 & -0.170965 & -5.507162 \\
\hline & -3.094811 & -2.220513 & -4.530204 \\
\hline & -3.920540 & 1.911579 & -4.449542 \\
\hline $\mathrm{H}$ & -2.529110 & -2.674881 & -2.353981 \\
\hline $\mathrm{H}$ & -3.416455 & 2.478513 & -2.253063 \\
\hline $\mathrm{H}$ & -2.965064 & -1.185775 & 0.70662 \\
\hline & -2.988568 & 1.199726 & 0.65387 \\
\hline & -2.242562 & 2.434321 & -0.36392 \\
\hline & -2.189721 & -2.498219 & -0.20108 \\
\hline
\end{tabular}

\section{Structure of 3:}
C
$\begin{array}{lll}0.152190 & -1.585344 & -1.465769\end{array}$
$\begin{array}{lllll}\text { C } & & 0.152191 & 1.585344 & -1.465768\end{array}$
$\begin{array}{llll}\text { C } & -0.537177 & -1.256226 & -2.631096\end{array}$ 


$\begin{array}{lrrr}\mathrm{C} & -0.537174 & 1.256227 & -2.631096 \\ \mathrm{C} & -0.858116 & 0.000001 & -3.152611 \\ \mathrm{C} & 0.664550 & -0.714710 & -0.497539 \\ \mathrm{C} & 0.664551 & 0.714710 & -0.497539 \\ \mathrm{C} & -1.243928 & -1.161788 & 2.021162 \\ \mathrm{C} & -1.243928 & 1.161789 & 2.021162 \\ \mathrm{C} & -2.009588 & 0.000001 & 2.297761 \\ \mathrm{C} & 1.237761 & 1.146674 & 0.843813 \\ \mathrm{C} & 1.237760 & -1.146676 & 0.843813 \\ \mathrm{C} & 0.035931 & 0.717636 & 1.658124 \\ \mathrm{C} & 0.035930 & -0.717636 & 1.658123 \\ \mathrm{H} & -1.581482 & -2.182985 & 2.131404 \\ \mathrm{H} & -1.581481 & 2.182986 & 2.131405 \\ \mathrm{H} & -3.014492 & 0.000002 & 2.700922 \\ \mathrm{H} & -1.403858 & 0.000001 & -4.090091 \\ \mathrm{H} & -0.858504 & 2.102809 & -3.232068 \\ \mathrm{H} & -0.858507 & -2.102808 & -3.232069 \\ \mathrm{H} & 0.308496 & 2.644246 & -1.282973 \\ \mathrm{H} & 0.308493 & -2.644246 & -1.282973 \\ \mathrm{C} & 2.235197 & -0.000001 & 1.140158 \\ \mathrm{H} & 1.592218 & -2.173466 & 0.906418 \\ \mathrm{H} & 1.592220 & 2.173464 & 0.906418 \\ \mathrm{H} & 3.095830 & -0.000002 & 0.463974 \\ \mathrm{H} & 2.565953 & -0.000002 & 2.180548\end{array}$

\section{Structure of 4:}

$\begin{array}{lrrr}\mathrm{C} & 0.781171 & 0.000000 & 0.000000 \\ \mathrm{C} & -0.390584 & -0.709221 & 0.000001 \\ \mathrm{C} & -0.390584 & 0.709221 & 0.000001 \\ \mathrm{C} & -1.017027 & -1.156860 & -2.974035 \\ \mathrm{C} & -1.017026 & 1.156862 & -2.974035 \\ \mathrm{C} & -0.658436 & 0.000001 & -3.706125 \\ \mathrm{C} & -1.797167 & 1.170714 & -0.387655 \\ \mathrm{C} & -1.797168 & -1.170713 & -0.387655 \\ \mathrm{C} & -1.690957 & 0.716139 & -1.821347 \\ \mathrm{C} & -1.690958 & -0.716136 & -1.821348 \\ \mathrm{H} & -0.845453 & -2.179011 & -3.282804 \\ \mathrm{H} & -0.845451 & 2.179013 & -3.282804 \\ \mathrm{H} & -0.224677 & 0.000001 & -4.698064 \\ \mathrm{C} & -2.623928 & 0.000001 & 0.239141 \\ \mathrm{H} & -2.070687 & -2.195223 & -0.154753 \\ \mathrm{H} & -2.070686 & 2.195226 & -0.154752 \\ \mathrm{H} & -2.606480 & 0.000001 & 1.332472 \\ \mathrm{H} & -3.652292 & 0.000002 & -0.123895 \\ \mathrm{H} & 1.857614 & 0.000000 & -0.072963\end{array}$

Page 4 of 22 


$\begin{array}{llll}\text { Structure of 5: } & & & \\ \mathrm{C} & -1.43546136 & 0.52898334 & -1.15262829 \\ \mathrm{C} & -1.43546136 & 0.52898334 & 1.15262829 \\ \mathrm{C} & 1.02733249 & -0.30523290 & -1.54450417 \\ \mathrm{C} & 1.02733249 & -0.30523290 & 1.54450417 \\ \mathrm{C} & 1.32813995 & 0.78642317 & -0.69487500 \\ \mathrm{C} & 1.32813995 & 0.78642317 & 0.69487500 \\ \mathrm{C} & 1.31346241 & -1.64335088 & -1.24626363 \\ \mathrm{C} & 1.31346241 & -1.64335088 & 1.24626363 \\ \mathrm{C} & -1.52834645 & -0.28923553 & 0.00000000 \\ \mathrm{C} & -1.32769834 & 1.86686692 & -0.70796074 \\ \mathrm{C} & -1.32769834 & 1.86686692 & 0.70796074 \\ \mathrm{C} & 1.56354584 & -2.21636792 & 0.00000000 \\ \mathrm{H} & 1.44828138 & 1.74895271 & -1.17732477 \\ \mathrm{H} & 1.44828138 & 1.74895271 & 1.17732477 \\ \mathrm{C} & 0.34647850 & 0.02154668 & -2.85915494 \\ \mathrm{C} & 0.34647850 & 0.02154668 & 2.85915494 \\ \mathrm{C} & -1.19179799 & 0.03069574 & -2.55985926 \\ \mathrm{C} & -1.19179799 & 0.03069574 & 2.55985926 \\ \mathrm{H} & 0.65651991 & 1.01148815 & -3.20439001 \\ \mathrm{H} & 0.65651991 & 1.01148815 & 3.20439001 \\ \mathrm{H} & -1.70519114 & 0.64057026 & -3.31058943 \\ \mathrm{H} & -1.70519114 & 0.64057026 & 3.31058943 \\ \mathrm{H} & 0.59980522 & -0.70055694 & -3.64158282 \\ \mathrm{H} & 0.59980522 & -0.70055694 & 3.64158282 \\ \mathrm{H} & -1.57798117 & -0.98797509 & -2.66066952 \\ \mathrm{H} & -1.57798117 & -0.98797509 & 2.66066952 \\ \mathrm{H} & 1.80362276 & -3.27546699 & 0.00000000 \\ \mathrm{H} & 1.27731683 & -2.33159491 & -2.08692314 \\ \mathrm{H} & 1.27731683 & -2.33159491 & 2.08692314 \\ \mathrm{H} & -1.25931022 & 2.74211768 & -1.34231766 \\ \mathrm{H} & -1.25931022 & 2.74211768 & 1.34231766 \\ \mathrm{H} & -1.61916856 & -1.36810067 & 0.00000000\end{array}$

Structure of 6:

$\begin{array}{lrrr}\mathrm{C} & -1.42261 & 0.17576 & 1.15256 \\ \mathrm{C} & -1.42261 & 0.17576 & -1.15256 \\ \mathrm{C} & 1.18261 & -0.44286 & 1.54449 \\ \mathrm{C} & 1.18261 & -0.44286 & -1.54449 \\ \mathrm{C} & 1.65315 & -1.44734 & 0.70470 \\ \mathrm{C} & 1.65315 & -1.44734 & -0.70470 \\ \mathrm{C} & 1.18112 & 0.94231 & 1.23418 \\ \mathrm{C} & 1.18112 & 0.94231 & -1.23418 \\ \mathrm{C} & -1.28710 & -0.62448 & 0.00000 \\ \mathrm{C} & -1.72970 & 1.50069 & 0.69929 \\ \mathrm{C} & -1.72970 & 1.50069 & -0.69929\end{array}$

Page 5 of 22 


$\begin{array}{lrrr}\mathrm{C} & 1.31012 & 1.56030 & 0.00000 \\ \mathrm{H} & 1.77432 & -2.42731 & 1.15928 \\ \mathrm{H} & 1.77432 & -2.42731 & -1.15928 \\ \mathrm{H} & -1.89427 & 2.35934 & 1.33783 \\ \mathrm{H} & -1.89427 & 2.35934 & -1.33783 \\ \mathrm{C} & 0.39536 & -0.77232 & 2.78321 \\ \mathrm{C} & 0.39536 & -0.77232 & -2.78321 \\ \mathrm{C} & -1.13051 & -0.30311 & 2.55190 \\ \mathrm{C} & -1.13051 & -0.30311 & -2.55190 \\ \mathrm{H} & 0.44081 & -1.84022 & 3.00403 \\ \mathrm{H} & 0.44081 & -1.84022 & -3.00403 \\ \mathrm{H} & -1.76723 & -1.15389 & 2.81263 \\ \mathrm{H} & -1.76723 & -1.15389 & -2.81263 \\ \mathrm{H} & 0.78044 & -0.23994 & 3.65772 \\ \mathrm{H} & 0.78044 & -0.23994 & -3.65772 \\ \mathrm{H} & -1.35981 & 0.48915 & 3.26840 \\ \mathrm{H} & -1.35981 & 0.48915 & -3.26840 \\ \mathrm{H} & 1.31350 & 2.64489 & 0.00000 \\ \mathrm{H} & 1.06019 & 1.60636 & 2.08479 \\ \mathrm{H} & 1.06019 & 1.60636 & -2.08479 \\ \mathrm{H} & -1.07352 & -1.68431 & 0.00000\end{array}$

\begin{tabular}{lrrr}
\multicolumn{4}{c}{ Structure of 5Ts: } \\
$\mathrm{C}$ & -1.910537 & 0.206979 & 0.752951 \\
$\mathrm{C}$ & -1.576930 & 0.545973 & -1.487394 \\
$\mathrm{C}$ & 0.942108 & -0.269553 & 1.661156 \\
$\mathrm{C}$ & 1.103160 & 0.108838 & -1.480879 \\
$\mathrm{C}$ & 0.814259 & -1.266484 & 0.615032 \\
$\mathrm{C}$ & 0.850970 & -1.083797 & -0.732383 \\
$\mathrm{C}$ & 1.777164 & 0.857440 & 1.501111 \\
$\mathrm{C}$ & 1.893524 & 1.135568 & -0.999769 \\
$\mathrm{C}$ & -1.417938 & 1.113718 & -0.226799 \\
$\mathrm{C}$ & -2.451352 & -0.931611 & 0.025527 \\
$\mathrm{C}$ & -2.270938 & -0.710106 & -1.323184 \\
$\mathrm{C}$ & 2.280718 & 1.402390 & 0.330754 \\
$\mathrm{H}$ & 0.532972 & -2.257846 & 0.956207 \\
$\mathrm{H}$ & 0.539579 & -1.938444 & -1.326297 \\
$\mathrm{C}$ & 0.081708 & -0.402910 & 2.776659 \\
$\mathrm{C}$ & 0.482544 & 0.227821 & -2.870360 \\
$\mathrm{C}$ & -1.677985 & 0.308874 & 2.147834 \\
$\mathrm{C}$ & -0.863879 & 0.997908 & -2.743945 \\
$\mathrm{H}$ & -0.250831 & -1.406098 & 3.020746 \\
$\mathrm{H}$ & 0.295523 & -0.772818 & -3.270677 \\
$\mathrm{H}$ & -2.280504 & -0.316717 & 2.800782 \\
$\mathrm{H}$ & -1.465178 & 0.842960 & -3.646687 \\
$\mathrm{H}$ & 0.283438 & 0.216027 & 3.646702
\end{tabular}

Page 6 of 22 


$\begin{array}{lrrr}\mathrm{H} & 1.160409 & 0.738166 & -3.563575 \\ \mathrm{H} & -1.529354 & 1.312702 & 2.534876 \\ \mathrm{H} & -0.656015 & 2.070993 & -2.677846 \\ \mathrm{H} & 2.937756 & 2.257861 & 0.457581 \\ \mathrm{H} & 2.023738 & 1.392945 & 2.415022 \\ \mathrm{H} & 2.200796 & 1.884613 & -1.726324 \\ \mathrm{H} & -2.937740 & -1.785434 & 0.480621 \\ \mathrm{H} & -2.580986 & -1.364031 & -2.128410 \\ \mathrm{H} & -0.904054 & 2.041743 & -0.011657\end{array}$

\section{Structure of 5PDT:}

\begin{tabular}{|c|c|c|c|}
\hline & -0.143761 & 3.117880 & 1.332828 \\
\hline 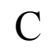 & -1.535589 & 1.715112 & 0.164333 \\
\hline C & 2.295888 & -2.310444 & -0.215931 \\
\hline C & -0.775381 & -1.275629 & -0.750605 \\
\hline $\mathrm{C}$ & 1.334752 & -1.626517 & 0.650246 \\
\hline $\mathrm{C}$ & 0.072401 & -1.195717 & 0.430345 \\
\hline $\mathrm{C}$ & 1.963750 & -2.670524 & -1.593380 \\
\hline C & -0.419749 & -1.848041 & -1.929155 \\
\hline $\mathrm{C}$ & -0.422825 & 2.477908 & 0.039157 \\
\hline $\mathrm{C}$ & -1.207591 & 2.666504 & 2.248891 \\
\hline $\mathrm{C}$ & -2.024947 & 1.842111 & 1.558642 \\
\hline $\mathrm{C}$ & 0.828368 & -2.475215 & -2.301151 \\
\hline $\mathrm{H}$ & 1.720955 & -1.433056 & 1.648151 \\
\hline $\mathrm{H}$ & -0.396070 & -0.707258 & 1.279647 \\
\hline $\mathrm{C}$ & 3.521745 & -2.619139 & 0.274820 \\
\hline $\mathrm{C}$ & -2.155918 & -0.658863 & -0.628821 \\
\hline $\mathrm{C}$ & 0.870552 & 3.945378 & 1.630691 \\
\hline $\mathrm{C}$ & -2.201757 & 0.874943 & -0.882009 \\
\hline $\mathrm{H}$ & 3.802977 & -2.369521 & 1.290559 \\
\hline $\mathrm{H}$ & -2.567588 & -0.871408 & 0.363913 \\
\hline $\mathrm{H}$ & 0.986245 & 4.363117 & 2.624835 \\
\hline $\mathrm{H}$ & -3.259017 & 1.162771 & -0.946284 \\
\hline $\mathrm{H}$ & 4.261926 & -3.126317 & -0.331997 \\
\hline $\mathrm{H}$ & -2.824137 & -1.133321 & -1.352315 \\
\hline $\mathrm{H}$ & 1.610341 & 4.224621 & 0.888505 \\
\hline $\mathrm{H}$ & -1.756494 & 1.083754 & -1.858863 \\
\hline $\mathrm{H}$ & 0.846052 & -2.850804 & -3.320918 \\
\hline $\mathrm{H}$ & 2.768637 & -3.178398 & -2.118815 \\
\hline $\mathrm{H}$ & -1.169344 & -1.843194 & -2.716588 \\
\hline 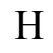 & -1.296031 & 2.969029 & 3.282924 \\
\hline$\pi$ & -2.909954 & 1.349816 & 1.942514 \\
\hline & 0.181866 & 2.601680 & -0.848674 \\
\hline
\end{tabular}

Structure of 7:

$\begin{array}{llll}\mathrm{N} & 0.20716 & -0.00000 & 0.00000\end{array}$ 


$\begin{array}{rrrr}\mathrm{C} & 0.72494 & 1.42215 & 0.00000 \\ \mathrm{C} & 0.72494 & -0.71107 & -1.23161 \\ \mathrm{C} & 0.72494 & -0.71107 & 1.23161 \\ \mathrm{H} & 1.81671 & 1.41440 & 0.00000 \\ \mathrm{H} & 1.81671 & -0.70720 & -1.22490 \\ \mathrm{H} & 1.81671 & -0.70720 & 1.22490 \\ \mathrm{H} & 0.33415 & 1.91346 & -0.88744 \\ \mathrm{H} & 0.33415 & 1.91346 & 0.88744 \\ \mathrm{H} & 0.33415 & -1.72528 & -1.21339 \\ \mathrm{H} & 0.33415 & -1.72528 & 1.21339 \\ \mathrm{H} & 0.33415 & -0.18818 & 2.10083 \\ \mathrm{H} & 0.33415 & -0.18818 & -2.10083 \\ \mathrm{C} & -1.20847 & 0.00000 & 0.00000 \\ \mathrm{C} & -2.45055 & 0.00000 & 0.00000\end{array}$

Structure of 7-H:

$\begin{array}{lrrr}\mathrm{N} & 0.00000 & 0.00000 & 0.27682 \\ \mathrm{C} & 1.43961 & 0.00000 & 0.78302 \\ \mathrm{C} & -0.71981 & -1.24674 & 0.78302 \\ \mathrm{C} & -0.71981 & 1.24674 & 0.78302 \\ \mathrm{H} & 1.41195 & 0.00000 & 1.87129 \\ \mathrm{H} & -0.70597 & -1.22278 & 1.87129 \\ \mathrm{H} & -0.70597 & 1.22278 & 1.87129 \\ \mathrm{H} & 1.93159 & -0.89338 & 0.40545 \\ \mathrm{H} & 1.93159 & 0.89338 & 0.40545 \\ \mathrm{H} & -1.73949 & -1.22612 & 0.40545 \\ \mathrm{H} & -1.73949 & 1.22612 & 0.40545 \\ \mathrm{H} & -0.19211 & 2.11950 & 0.40545 \\ \mathrm{H} & -0.19211 & -2.11950 & 0.40545 \\ \mathrm{C} & 0.00000 & 0.00000 & -1.12711 \\ \mathrm{C} & 0.00000 & 0.00000 & -2.32134 \\ \mathrm{H} & 0.00000 & 0.00000 & -3.38792\end{array}$

Structure of Acetylene (C2H2):

$\begin{array}{rrrr}\mathrm{C} & 0.00000 & -0.00000 & 0.59966 \\ \mathrm{C} & 0.00000 & 0.00000 & -0.59966 \\ \mathrm{H} & 0.00000 & 0.00000 & -1.66284 \\ \mathrm{H} & 0.00000 & -0.00000 & 1.66284\end{array}$

Structure of Acetylene anion (C2H-):
$\begin{array}{llll}\text { C } & -2.26244 & -0.26313 & 0.00024\end{array}$
$\begin{array}{llll}\text { C } & -1.14904 & 0.30530 & -0.00011\end{array}$
H $\quad-3.22938 \quad-0.75543 \quad 0.00176$ 


\section{Structure of 8a:}

$\begin{array}{lccc}\mathrm{N} & 0.02179 & -1.31092 & 0.01360 \\ \mathrm{C} & -0.00314 & 0.24888 & -0.01063 \\ \mathrm{C} & -1.29626 & 0.71143 & -0.01586 \\ \mathrm{C} & 1.27578 & 0.75051 & 0.00478 \\ \mathrm{H} & -2.11136 & 0.12265 & -0.42345 \\ \mathrm{H} & 2.10680 & 0.18238 & 0.40910 \\ \mathrm{H} & -1.48956 & 1.75716 & 0.17519 \\ \mathrm{H} & 1.43010 & 1.80936 & -0.14714 \\ \mathrm{H} & 0.93236 & -1.63797 & -0.32501 \\ \mathrm{H} & -0.17061 & -1.66181 & 0.95325 \\ \mathrm{H} & -0.70859 & -1.66021 & -0.60689\end{array}$

\section{Structure of 8b:}

$\begin{array}{llll}\mathrm{C} & -0.31045687 & -0.67999161 & 0.16396929 \\ \mathrm{C} & -0.03699427 & 0.72136343 & 0.27805280 \\ \mathrm{C} & -1.47653875 & -1.29546480 & -0.15143832 \\ \mathrm{H} & 0.51467163 & -1.33969790 & 0.45936307 \\ \mathrm{~N} & 1.39480310 & 1.03295243 & -0.12427694 \\ \mathrm{H} & -0.68760293 & 1.39925784 & -0.27193901 \\ \mathrm{H} & -1.59032652 & -2.36719411 & -0.04816025 \\ \mathrm{H} & -2.35729847 & -0.73086193 & -0.44061203 \\ \mathrm{H} & 1.61584193 & 1.99647592 & 0.11829971 \\ \mathrm{H} & 2.01707975 & 0.44508764 & 0.43007617 \\ \mathrm{H} & 1.66795220 & 0.89082337 & -1.12059168\end{array}$

\begin{tabular}{lrrc}
\multicolumn{4}{c}{ Structure of Allyl anion: } \\
H & 0.00000 & 0.00000 & 0.00000 \\
$\mathrm{C}$ & 1.09767 & 0.00000 & 0.00000 \\
$\mathrm{C}$ & 1.66295 & 1.27438 & 0.00000 \\
$\mathrm{C}$ & 1.66295 & -1.27438 & -0.00000 \\
$\mathrm{H}$ & 1.03890 & 2.16232 & 0.00000 \\
$\mathrm{H}$ & 2.74048 & 1.42531 & -0.00000 \\
$\mathrm{H}$ & 1.03890 & -2.16232 & -0.00000 \\
$\mathrm{H}$ & 2.74048 & -1.42531 & -0.00000
\end{tabular}

\section{Structure of 9:}

$\begin{array}{lrrc}\mathrm{C} & 0.00000 & 0.09051 & -0.34789 \\ \mathrm{C} & -1.15773 & -0.00112 & -1.15268 \\ \mathrm{C} & 1.15773 & -0.00112 & -1.15268 \\ \mathrm{C} & -0.70422 & -0.10953 & -2.48000 \\ \mathrm{C} & 0.70422 & -0.10953 & -2.48000 \\ \mathrm{~N} & 0.00000 & 0.04470 & 1.14260 \\ \mathrm{H} & -2.19241 & 0.02978 & -0.84096 \\ \mathrm{H} & 2.19241 & 0.02978 & -0.84096 \\ \mathrm{H} & -1.34165 & -0.15519 & -3.35277\end{array}$




$\begin{array}{rrrr}\mathrm{H} & 1.34165 & -0.15519 & -3.35277 \\ \mathrm{C} & 0.00000 & -1.38784 & 1.62332 \\ \mathrm{C} & 1.21410 & 0.73611 & 1.69091 \\ \mathrm{C} & -1.21410 & 0.73611 & 1.69091 \\ \mathrm{H} & -1.18034 & 0.70490 & 2.78091 \\ \mathrm{H} & 1.18034 & 0.70490 & 2.78091 \\ \mathrm{H} & -1.21489 & 1.76141 & 1.32936 \\ \mathrm{H} & 1.21489 & 1.76141 & 1.32936 \\ \mathrm{H} & -2.10086 & 0.22305 & 1.33050 \\ \mathrm{H} & 2.10086 & 0.22305 & 1.33050 \\ \mathrm{H} & 0.00000 & -1.41837 & 2.71600 \\ \mathrm{H} & -0.88608 & -1.87197 & 1.22020 \\ \mathrm{H} & 0.88608 & -1.87197 & 1.22020\end{array}$

\section{Structure of Cyclopentadienyl anion:}

$\begin{array}{lrrc}\mathrm{C} & 0.00000 & 0.26100 & 1.17518 \\ \mathrm{C} & 0.00000 & -1.03701 & 0.61138 \\ \mathrm{C} & 0.00000 & 1.19832 & 0.11492 \\ \mathrm{C} & 0.00000 & -0.90191 & -0.79732 \\ \mathrm{C} & 0.00000 & 0.47960 & -1.10415 \\ \mathrm{H} & 0.00000 & 0.49645 & 2.23526 \\ \mathrm{H} & 0.00000 & -1.97245 & 1.16288 \\ \mathrm{H} & 0.00000 & 2.27927 & 0.21858 \\ \mathrm{H} & 0.00000 & -1.71549 & -1.51656 \\ \mathrm{H} & 0.00000 & 0.91222 & -2.10017\end{array}$

Structure of 9-TS:

$\begin{array}{lrrc}\mathrm{C} & -0.18519 & -0.04175 & -0.18411 \\ \mathrm{C} & -1.19739 & 0.94570 & -0.36170 \\ \mathrm{C} & -0.76773 & -1.33921 & -0.27573 \\ \mathrm{C} & -2.40629 & 0.25502 & -0.45000 \\ \mathrm{C} & -2.14350 & -1.14246 & -0.39742 \\ \mathrm{~N} & 1.25309 & 0.23940 & 0.10013 \\ \mathrm{H} & -1.06839 & 2.01767 & -0.36601 \\ \mathrm{H} & -0.25862 & -2.28865 & -0.20397 \\ \mathrm{H} & -3.38053 & 0.71043 & -0.56330 \\ \mathrm{H} & -2.88455 & -1.92718 & -0.46405 \\ \mathrm{C} & 0.39043 & 0.14184 & 1.81937 \\ \mathrm{C} & 2.14789 & -0.84855 & -0.27133 \\ \mathrm{C} & 1.69726 & 1.54782 & -0.36143 \\ \mathrm{H} & 2.75390 & 1.68225 & -0.12495 \\ \mathrm{H} & 3.17799 & -0.57299 & -0.04015 \\ \mathrm{H} & 1.53781 & 1.64346 & -1.44501 \\ \mathrm{H} & 2.04880 & -1.07384 & -1.34284 \\ \mathrm{H} & 1.12008 & 2.33121 & 0.13223 \\ \mathrm{H} & 1.88753 & -1.74993 & 0.28568\end{array}$

Page 10 of 22 


$\begin{array}{crcc}\mathrm{H} & 1.03930 & -0.64956 & 2.18228 \\ \mathrm{H} & 0.70305 & 1.13878 & 2.11504 \\ \mathrm{H} & -0.66091 & -0.04798 & 2.02827\end{array}$

\section{Structure of 9-PDT:}

$\begin{array}{lrrr}\mathrm{C} & -0.41512 & 0.16262 & -0.04735 \\ \mathrm{C} & -0.17604 & 0.94020 & 1.24166 \\ \mathrm{C} & 0.62698 & 0.79593 & -0.97487 \\ \mathrm{C} & 0.76018 & 1.88104 & 1.04902 \\ \mathrm{C} & 1.26867 & 1.78494 & -0.33325 \\ \mathrm{~N} & -0.29724 & -1.29880 & 0.17148 \\ \mathrm{H} & -0.74177 & 0.74900 & 2.14404 \\ \mathrm{H} & 0.77445 & 0.49625 & -2.00401 \\ \mathrm{H} & 1.09584 & 2.60954 & 1.77626 \\ \mathrm{H} & 2.03338 & 2.42964 & -0.74912 \\ \mathrm{C} & -1.83537 & 0.51729 & -0.55478 \\ \mathrm{C} & -0.61205 & -2.12370 & -0.99091 \\ \mathrm{C} & 0.98559 & -1.70343 & 0.74533 \\ \mathrm{H} & 0.92476 & -2.75457 & 1.03907 \\ \mathrm{H} & -0.55544 & -3.17422 & -0.69496 \\ \mathrm{H} & 1.83170 & -1.59287 & 0.04653 \\ \mathrm{H} & 0.08260 & -1.97974 & -1.83778 \\ \mathrm{H} & 1.20137 & -1.11327 & 1.63606 \\ \mathrm{H} & -1.62694 & -1.93929 & -1.34198 \\ \mathrm{H} & -2.58466 & 0.06769 & 0.10168 \\ \mathrm{H} & -2.00502 & 0.16334 & -1.57316 \\ \mathrm{H} & -1.96665 & 1.60077 & -0.55195\end{array}$

\section{Structure of 10:}

$\begin{array}{lrrr}\mathrm{C} & 0.00000 & 0.00000 & 0.99278 \\ \mathrm{C} & -1.15191 & 0.15947 & 1.82828 \\ \mathrm{C} & 1.15191 & -0.15947 & 1.82828 \\ \mathrm{C} & -0.70970 & 0.10372 & 3.13726 \\ \mathrm{C} & 0.70970 & -0.10372 & 3.13726 \\ \mathrm{~N} & 0.00000 & 0.00000 & -0.39449 \\ \mathrm{H} & -2.16650 & 0.35171 & 1.51208 \\ \mathrm{H} & 2.16650 & -0.35171 & 1.51208 \\ \mathrm{H} & -1.33000 & 0.21361 & 4.01584 \\ \mathrm{H} & 1.33000 & -0.21361 & 4.01584 \\ \mathrm{C} & -1.17051 & -0.12383 & -1.11263 \\ \mathrm{C} & 1.17051 & 0.12383 & -1.11263 \\ \mathrm{C} & -1.18503 & -0.11170 & -2.48854 \\ \mathrm{C} & 1.18503 & 0.11170 & -2.48854 \\ \mathrm{C} & 0.00000 & 0.00000 & -3.21754 \\ \mathrm{H} & -2.06314 & -0.25420 & -0.52370 \\ \mathrm{H} & 2.06314 & 0.25420 & -0.52370\end{array}$

Page 11 of 22 


$\begin{array}{llll}\mathrm{H} & -2.14193 & -0.21053 & -2.98592 \\ \mathrm{H} & 2.14193 & 0.21053 & -2.98592 \\ \mathrm{H} & 0.00000 & 0.00000 & -4.29900\end{array}$

\section{Structure of 11:}

$\begin{array}{lrrl}\mathrm{N} & -0.65855 & 0.00000 & 0.00000 \\ \mathrm{C} & 0.04131 & 1.18276 & 0.00000 \\ \mathrm{C} & 0.04131 & -1.18276 & 0.00000 \\ \mathrm{C} & 1.41899 & 1.19667 & 0.00000 \\ \mathrm{C} & 1.41899 & -1.19667 & 0.00000 \\ \mathrm{C} & 2.13542 & 0.00000 & 0.00000 \\ \mathrm{C} & -2.01110 & 0.00000 & 0.00000 \\ \mathrm{C} & -3.26223 & 0.00000 & 0.00000 \\ \mathrm{H} & -0.57575 & 2.06875 & 0.00000 \\ \mathrm{H} & -0.57575 & -2.06875 & 0.00000 \\ \mathrm{H} & 1.92381 & 2.15426 & 0.00000 \\ \mathrm{H} & 1.92381 & -2.15426 & 0.00000 \\ \mathrm{H} & 3.21754 & 0.00000 & 0.00000\end{array}$

\section{Structure of 11-H:}

$\begin{array}{rrrr}\mathrm{N} & 0.00000 & 0.00000 & -0.56371 \\ \mathrm{C} & 0.00000 & 1.19141 & 0.10848 \\ \mathrm{C} & 0.00000 & -1.19141 & 0.10848 \\ \mathrm{C} & 0.00000 & 1.20569 & 1.48596 \\ \mathrm{C} & 0.00000 & -1.20569 & 1.48596 \\ \mathrm{C} & 0.00000 & 0.00000 & 2.18882 \\ \mathrm{C} & 0.00000 & 0.00000 & -1.93325 \\ \mathrm{C} & 0.00000 & 0.00000 & -3.12969 \\ \mathrm{H} & 0.00000 & 2.07939 & -0.50777 \\ \mathrm{H} & 0.00000 & -2.07939 & -0.50777 \\ \mathrm{H} & 0.00000 & 2.15891 & 1.99834 \\ \mathrm{H} & 0.00000 & -2.15891 & 1.99834 \\ \mathrm{H} & 0.00000 & 0.00000 & 3.27223 \\ \mathrm{H} & 0.00000 & 0.00000 & -4.19601\end{array}$

\section{Structure of 12:}

$\begin{array}{lrrl}\mathrm{C} & -0.00478 & -2.12957 & 0.16508 \\ \mathrm{C} & 0.00475 & 2.12957 & 0.16508 \\ \mathrm{C} & -1.18333 & -1.34463 & 0.10209 \\ \mathrm{C} & 1.18332 & 1.34463 & 0.10228 \\ \mathrm{C} & -1.17654 & 1.34972 & 0.10741 \\ \mathrm{C} & 1.17652 & -1.34972 & 0.10759 \\ \mathrm{C} & -0.72321 & 0.00147 & 0.05402 \\ \mathrm{C} & 0.72320 & -0.00147 & 0.05413 \\ \mathrm{~N} & -0.00028 & -3.61480 & -0.02534 \\ \mathrm{~N} & 0.00029 & 3.61480 & -0.02534\end{array}$

Page 12 of 22 


$\begin{array}{lrrr}\mathrm{H} & -2.20956 & -1.68025 & 0.14462 \\ \mathrm{H} & 2.20954 & 1.68025 & 0.14497 \\ \mathrm{H} & -2.20184 & 1.69171 & 0.13394 \\ \mathrm{H} & 2.20182 & -1.69171 & 0.13429 \\ \mathrm{C} & 0.11652 & -3.95064 & -1.49355 \\ \mathrm{C} & -0.11629 & 3.95064 & -1.49357 \\ \mathrm{C} & -1.15401 & 4.23279 & 0.70620 \\ \mathrm{C} & 1.15390 & -4.23279 & 0.70638 \\ \mathrm{C} & 1.26373 & 4.22113 & 0.50480 \\ \mathrm{C} & -1.26381 & -4.22113 & 0.50460 \\ \mathrm{H} & -1.21656 & -5.30462 & 0.38497 \\ \mathrm{H} & 1.21650 & 5.30462 & 0.38516 \\ \mathrm{H} & -0.11084 & 5.03505 & -1.63662 \\ \mathrm{H} & 0.11110 & -5.03505 & -1.63660 \\ \mathrm{H} & 1.13756 & -5.31397 & 0.55481 \\ \mathrm{H} & -1.13765 & 5.31397 & 0.55463 \\ \mathrm{H} & -1.36091 & -3.94646 & 1.55211 \\ \mathrm{H} & 1.36066 & 3.94646 & 1.55232 \\ \mathrm{H} & 0.72464 & 3.48356 & -2.00089 \\ \mathrm{H} & -0.72433 & -3.48356 & -2.00100 \\ \mathrm{H} & 1.06055 & -3.98319 & 1.76040 \\ \mathrm{H} & -1.06083 & 3.98319 & 1.76023 \\ \mathrm{H} & 2.10249 & 3.81703 & -0.05464 \\ \mathrm{H} & -2.10249 & -3.81703 & -0.05497 \\ \mathrm{H} & -1.04163 & 3.51047 & -1.85671 \\ \mathrm{H} & 1.04192 & -3.51047 & -1.85654 \\ \mathrm{H} & 2.07654 & -3.81285 & 0.31764 \\ \mathrm{H} & -2.07659 & 3.81285 & 0.31732\end{array}$

Structure of Pentalene Dianion:

$\begin{array}{lrrr}\mathrm{C} & -2.18546 & 0.00000 & 0.00000 \\ \mathrm{C} & 0.00000 & 0.72385 & 0.00000 \\ \mathrm{C} & 0.00000 & -0.72385 & 0.00000 \\ \mathrm{C} & 2.18546 & 0.00000 & 0.00000 \\ \mathrm{C} & -1.36631 & 1.16714 & 0.00000 \\ \mathrm{C} & 1.36631 & 1.16714 & 0.00000 \\ \mathrm{C} & -1.36631 & -1.16714 & 0.00000 \\ \mathrm{C} & 1.36631 & -1.16714 & 0.00000 \\ \mathrm{H} & -3.27676 & 0.00000 & 0.00000 \\ \mathrm{H} & 3.27676 & 0.00000 & 0.00000 \\ \mathrm{H} & -1.73076 & 2.19366 & 0.00000 \\ \mathrm{H} & 1.73076 & 2.19366 & 0.00000 \\ \mathrm{H} & -1.73076 & -2.19366 & 0.00000 \\ \mathrm{H} & 1.73076 & -2.19366 & 0.00000\end{array}$

Page 13 of 22 


\section{Structure of 13a:}

\begin{tabular}{|c|c|c|c|}
\hline $\mathrm{C}$ & -0.03734 & 0.00000 & 1.85357 \\
\hline $\mathrm{C}$ & 0.03734 & 0.00000 & -1.85357 \\
\hline $\mathrm{C}$ & -1.32632 & 0.00000 & 1.29426 \\
\hline C & 1.32632 & 0.00000 & -1.29426 \\
\hline $\mathrm{C}$ & -1.26339 & 0.00000 & -1.32238 \\
\hline $\mathrm{C}$ & 1.26339 & 0.00000 & 1.32238 \\
\hline $\mathrm{C}$ & -1.80349 & 0.00000 & -0.02610 \\
\hline $\mathrm{C}$ & 1.80349 & 0.00000 & 0.02610 \\
\hline $\mathrm{N}$ & 0.00091 & 0.00000 & -3.42304 \\
\hline $\mathrm{N}$ & -0.00091 & 0.00000 & 3.42304 \\
\hline $\mathrm{H}$ & -2.15212 & 0.00000 & 1.98810 \\
\hline $\mathrm{H}$ & 2.15212 & 0.00000 & -1.98810 \\
\hline $\mathrm{H}$ & -2.06247 & 0.00000 & -2.05647 \\
\hline $\mathrm{H}$ & 2.06247 & 0.00000 & 2.05647 \\
\hline $\mathrm{C}$ & -0.70552 & 1.23412 & -3.92316 \\
\hline $\mathrm{C}$ & -0.70552 & -1.23412 & -3.92316 \\
\hline $\mathrm{C}$ & 0.70552 & 1.23412 & 3.92316 \\
\hline $\mathrm{C}$ & 0.70552 & -1.23412 & 3.92316 \\
\hline $\mathrm{C}$ & 1.34562 & 0.00000 & -4.08651 \\
\hline $\mathrm{C}$ & -1.34562 & 0.00000 & 4.08651 \\
\hline $\mathrm{H}$ & -0.72788 & -1.22682 & -5.01617 \\
\hline $\mathrm{H}$ & -0.72788 & 1.22682 & -5.01617 \\
\hline $\mathrm{H}$ & 0.72788 & -1.22682 & 5.01617 \\
\hline $\mathrm{H}$ & 0.72788 & 1.22682 & 5.01617 \\
\hline $\mathrm{H}$ & 1.18446 & 0.00000 & -5.16485 \\
\hline $\mathrm{H}$ & -1.18446 & 0.00000 & 5.16485 \\
\hline $\mathrm{H}$ & -0.15546 & -2.09718 & -3.55446 \\
\hline $\mathrm{H}$ & -0.15546 & 2.09718 & -3.55446 \\
\hline $\mathrm{H}$ & 0.15546 & -2.09718 & 3.55446 \\
\hline $\mathrm{H}$ & 0.15546 & 2.09718 & 3.55446 \\
\hline $\mathrm{H}$ & 1.89564 & -0.88990 & -3.79355 \\
\hline $\mathrm{H}$ & 1.89564 & 0.88990 & -3.79355 \\
\hline $\mathrm{H}$ & -1.89564 & -0.88990 & 3.79355 \\
\hline $\mathrm{H}$ & -1.89564 & 0.88990 & 3.79355 \\
\hline $\mathrm{H}$ & -1.71258 & -1.26110 & -3.52276 \\
\hline $\mathrm{H}$ & -1.71258 & 1.26110 & -3.52276 \\
\hline $\mathrm{H}$ & 1.71258 & -1.26110 & 3.52276 \\
\hline $\mathrm{H}$ & 1.71258 & 1.26110 & 3.52276 \\
\hline $\mathrm{H}$ & 2.89180 & 0.00000 & 0.05168 \\
\hline $\mathrm{H}$ & -2.89180 & 0.00000 & -0.05168 \\
\hline
\end{tabular}

\section{Structure of 13b:}
C $\quad-1.71883$
$-1.56399$
0.00000
$\begin{array}{llll}\text { C } & 1.71883 & -1.56399 & 0.00000\end{array}$
$\begin{array}{llll}\text { C } & 1.71234 & -0.15774 & 0.00000\end{array}$

Page 14 of 22 


$\begin{array}{lrrr}\mathrm{C} & -1.71234 & -0.15774 & 0.00000 \\ \mathrm{C} & 0.70324 & 0.82478 & 0.00000 \\ \mathrm{C} & -0.70324 & 0.82478 & 0.00000 \\ \mathrm{C} & -0.70146 & -2.52718 & 0.00000 \\ \mathrm{C} & 0.70146 & -2.52718 & 0.00000 \\ \mathrm{~N} & 3.13366 & 0.50775 & 0.00000 \\ \mathrm{~N} & -3.13366 & 0.50775 & 0.00000 \\ \mathrm{H} & 2.68748 & -2.03886 & 0.00000 \\ \mathrm{H} & -2.68748 & -2.03886 & 0.00000 \\ \mathrm{H} & 1.06160 & 1.84879 & 0.00000 \\ \mathrm{H} & -1.06160 & 1.84879 & 0.00000 \\ \mathrm{H} & -1.10898 & -3.53689 & 0.00000 \\ \mathrm{H} & 1.10898 & -3.53689 & 0.00000 \\ \mathrm{C} & 3.31299 & 1.35455 & 1.23378 \\ \mathrm{C} & -3.31299 & 1.35455 & 1.23378 \\ \mathrm{C} & -3.31299 & 1.35455 & -1.23378 \\ \mathrm{C} & 3.31299 & 1.35455 & -1.23378 \\ \mathrm{C} & 4.28000 & -0.46002 & 0.00000 \\ \mathrm{C} & -4.28000 & -0.46002 & 0.00000 \\ \mathrm{H} & 4.30471 & 1.81460 & -1.22382 \\ \mathrm{H} & -4.30471 & 1.81460 & -1.22382 \\ \mathrm{H} & -4.30471 & 1.81460 & 1.22382 \\ \mathrm{H} & 4.30471 & 1.81460 & 1.22382 \\ \mathrm{H} & 5.20392 & 0.11910 & 0.00000 \\ \mathrm{H} & -5.20392 & 0.11910 & 0.00000 \\ \mathrm{H} & 3.19907 & 0.70252 & -2.09697 \\ \mathrm{H} & -3.19907 & 0.70252 & -2.09697 \\ \mathrm{H} & -3.19907 & 0.70252 & 2.09697 \\ \mathrm{H} & 3.19907 & 0.70252 & 2.09697 \\ \mathrm{H} & 4.23106 & -1.08107 & -0.88987 \\ \mathrm{H} & -4.23106 & -1.08107 & -0.88987 \\ \mathrm{H} & -4.23106 & -1.08107 & 0.88987 \\ \mathrm{H} & 4.23106 & -1.08107 & 0.88987 \\ \mathrm{H} & 2.54192 & 2.11588 & -1.26382 \\ \mathrm{H} & -2.54192 & 2.11588 & -1.26382 \\ & -2.54192 & 2.11588 & 1.26382 \\ & 2.54192 & 2.11588 & 1.26382\end{array}$

Structure of Cyclooctateraene Dianion:
c
$\begin{array}{llll}\mathrm{C} & 1.308402 & 1.308402 & 0.000000\end{array}$
$\begin{array}{llll}\text { C } & 1.850359 & 0.000000 & 0.000000\end{array}$
$\begin{array}{llll}\text { C } & 1.308402 & -1.308402 & 0.000000\end{array}$
$\begin{array}{llll}\mathrm{C} & 0.000000 & -1.850359 & 0.000000\end{array}$
$\begin{array}{llll}\text { C } & -1.308402 & -1.308402 & 0.000000\end{array}$
$\begin{array}{llll}\text { C } & -1.850359 & 0.000000 & 0.000000\end{array}$

Page 15 of 22 


$\begin{array}{lrrr}\mathrm{C} & -1.308402 & 1.308402 & 0.000000 \\ \mathrm{H} & 0.000000 & 2.947075 & 0.000000 \\ \mathrm{H} & 2.083897 & 2.083897 & 0.000000 \\ \mathrm{H} & 2.947075 & 0.000000 & 0.000000 \\ \mathrm{H} & 2.083897 & -2.083897 & 0.000000 \\ \mathrm{H} & 0.000000 & -2.947075 & 0.000000 \\ \mathrm{H} & -2.083897 & -2.083897 & 0.000000 \\ \mathrm{H} & -2.947075 & 0.000000 & 0.000000 \\ \mathrm{H} & -2.083897 & 2.083897 & 0.000000\end{array}$

\section{Structure of 14:}

$\begin{array}{lrrr}\mathrm{C} & -1.183300 & 0.000000 & 0.000000 \\ \mathrm{C} & 0.961522 & -0.702585 & 0.000000 \\ \mathrm{C} & 0.961522 & 0.702585 & 0.000000 \\ \mathrm{~N} & 3.248238 & 0.000000 & 0.000000 \\ \mathrm{C} & -0.369872 & -1.157042 & 0.000000 \\ \mathrm{C} & -0.369872 & 1.157042 & 0.000000 \\ \mathrm{C} & 2.327505 & -1.268769 & 0.000000 \\ \mathrm{C} & 2.327505 & 1.268769 & 0.000000 \\ \mathrm{H} & -2.265858 & 0.000000 & 0.000000 \\ \mathrm{H} & -0.714579 & -2.182213 & 0.000000 \\ \mathrm{H} & -0.714579 & 2.182213 & 0.000000 \\ \mathrm{H} & 2.608976 & -1.856250 & 0.883811 \\ \mathrm{H} & 2.608976 & 1.856250 & 0.883811 \\ \mathrm{H} & 2.608976 & 1.856250 & -0.883811 \\ \mathrm{H} & 2.608976 & -1.856250 & -0.883811 \\ \mathrm{C} & 4.108537 & 0.000000 & 1.222371 \\ \mathrm{C} & 4.108537 & 0.000000 & -1.222371 \\ \mathrm{H} & 4.734483 & 0.892323 & -1.224419 \\ \mathrm{H} & 4.734483 & -0.892323 & -1.224419 \\ \mathrm{H} & 4.734483 & -0.892323 & 1.224419 \\ \mathrm{H} & 4.734483 & 0.892323 & 1.224419 \\ \mathrm{H} & 3.459039 & 0.000000 & -2.096083 \\ \mathrm{H} & 3.459039 & 0.000000 & 2.096083\end{array}$

\section{Structure of 15:}

$\begin{array}{lrrr}\mathrm{C} & 0.52862 & 0.00000 & -2.85090 \\ \mathrm{C} & -0.16053 & 0.70554 & -0.81854 \\ \mathrm{C} & -0.16053 & -0.70554 & -0.81854 \\ \mathrm{~N} & -0.16110 & 0.00000 & 1.43290 \\ \mathrm{C} & 0.27958 & 1.15720 & -2.07490 \\ \mathrm{C} & 0.27958 & -1.15720 & -2.07490 \\ \mathrm{C} & -0.49956 & 1.22585 & 0.52617 \\ \mathrm{C} & -0.49956 & -1.22585 & 0.52617 \\ \mathrm{H} & 0.86896 & 0.00000 & -3.87828 \\ \mathrm{H} & 0.39980 & 2.18217 & -2.39946\end{array}$

Page 16 of 22 


$\begin{array}{rrrr}\mathrm{H} & 0.39980 & -2.18217 & -2.39946 \\ \mathrm{H} & 0.07471 & 2.08321 & 0.89218 \\ \mathrm{H} & 0.07471 & -2.08321 & 0.89218 \\ \mathrm{H} & -1.56605 & -1.42290 & 0.71231 \\ \mathrm{H} & -1.56605 & 1.42290 & 0.71231 \\ \mathrm{C} & 1.30836 & 0.00000 & 1.72092 \\ \mathrm{C} & -0.93320 & 0.00000 & 2.70537 \\ \mathrm{H} & -0.68201 & -0.89113 & 3.28118 \\ \mathrm{H} & -0.68201 & 0.89113 & 3.28118 \\ \mathrm{H} & 1.55816 & 0.89256 & 2.29428 \\ \mathrm{H} & 1.55816 & -0.89256 & 2.29428 \\ \mathrm{H} & -1.99745 & 0.00000 & 2.47618 \\ \mathrm{H} & 1.83043 & 0.00000 & 0.76561\end{array}$

\section{Structure of 16:}

$\begin{array}{lrrc}\mathrm{C} & 0.96945 & -0.00000 & 0.00000 \\ \mathrm{~N} & -1.64400 & 0.00000 & 0.00000 \\ \mathrm{C} & 0.41037 & 1.44164 & 0.00000 \\ \mathrm{C} & 0.41037 & -0.72082 & -1.24850 \\ \mathrm{C} & 0.41037 & -0.72082 & 1.24850 \\ \mathrm{C} & -1.14966 & 1.38480 & 0.00000 \\ \mathrm{C} & -1.14966 & -0.69240 & -1.19927 \\ \mathrm{C} & -1.14966 & -0.69240 & 1.19927 \\ \mathrm{C} & 2.49860 & -0.00000 & 0.00000 \\ \mathrm{H} & 0.78233 & 1.97796 & -0.87962 \\ \mathrm{H} & 0.78233 & 1.97796 & 0.87962 \\ \mathrm{H} & 0.78233 & -1.75076 & -1.27315 \\ \mathrm{H} & 0.78233 & -1.75076 & 1.27315 \\ \mathrm{H} & 0.78233 & -0.22721 & 2.15278 \\ \mathrm{H} & 0.78233 & -0.22721 & -2.15278 \\ \mathrm{H} & -1.56080 & 1.88862 & -0.87908 \\ \mathrm{H} & -1.56080 & 1.88862 & 0.87908 \\ \mathrm{H} & -1.56080 & -1.70562 & -1.19605 \\ \mathrm{H} & -1.56080 & -1.70562 & 1.19605 \\ \mathrm{H} & -1.56080 & -0.18300 & 2.07513 \\ \mathrm{H} & -1.56080 & -0.18300 & -2.07513 \\ \mathrm{H} & 2.89255 & -1.02157 & 0.00000 \\ \mathrm{H} & 2.89255 & 0.51079 & 0.88471 \\ \mathrm{H} & 2.89255 & 0.51079 & -0.88471\end{array}$

\section{Structure of 17:}

$\begin{array}{llll}\mathrm{N} & 0.00000 & 0.00000 & 0.90465\end{array}$

$\begin{array}{llll}\mathrm{C} & 0.00000 & 0.00000 & -1.71656\end{array}$

$\begin{array}{llll}\mathrm{C} & -1.43829 & 0.00000 & 0.38501\end{array}$

$\begin{array}{llll}\text { C } & 0.71914 & 1.24559 & 0.38501\end{array}$

$\begin{array}{llll}\text { C } & 0.71914 & -1.24559 & 0.38501\end{array}$ 


$\begin{array}{lrrc}\mathrm{C} & -1.41929 & 0.00000 & -1.18415 \\ \mathrm{C} & 0.70965 & 1.22914 & -1.18415 \\ \mathrm{C} & 0.70965 & -1.22914 & -1.18415 \\ \mathrm{C} & 0.00000 & 0.00000 & 2.39141 \\ \mathrm{H} & -1.90457 & 0.88919 & 0.82180 \\ \mathrm{H} & -1.90457 & -0.88919 & 0.82180 \\ \mathrm{H} & 1.72234 & 1.20481 & 0.82180 \\ \mathrm{H} & 1.72234 & -1.20481 & 0.82180 \\ \mathrm{H} & 0.18223 & -2.09400 & 0.82180 \\ \mathrm{H} & 0.18223 & 2.09400 & 0.82180 \\ \mathrm{H} & -1.96991 & 0.88267 & -1.52840 \\ \mathrm{H} & -1.96991 & -0.88267 & -1.52840 \\ \mathrm{H} & 1.74937 & 1.26466 & -1.52840 \\ \mathrm{H} & 1.74937 & -1.26466 & -1.52840 \\ \mathrm{H} & 0.22054 & -2.14733 & -1.52840 \\ \mathrm{H} & 0.22054 & 2.14733 & -1.52840 \\ \mathrm{H} & 1.03075 & 0.00000 & 2.74747 \\ \mathrm{H} & -0.51538 & -0.89266 & 2.74747 \\ \mathrm{H} & -0.51538 & 0.89266 & 2.74747\end{array}$

Structure of 17-H:

$\begin{array}{lrrr}\mathrm{N} & -0.00047 & 0.00008 & 0.93345 \\ \mathrm{C} & 0.00075 & 0.00001 & -1.65297 \\ \mathrm{C} & -0.08152 & 1.42880 & 0.41010 \\ \mathrm{C} & 1.27754 & -0.64408 & 0.41125 \\ \mathrm{C} & -1.19710 & -0.78485 & 0.40987 \\ \mathrm{C} & 0.08037 & 1.44271 & -1.12575 \\ \mathrm{C} & 1.21042 & -0.79033 & -1.12488 \\ \mathrm{C} & -1.28898 & -0.65237 & -1.12624 \\ \mathrm{C} & -0.00097 & 0.00001 & 2.43058 \\ \mathrm{H} & 0.69828 & 1.99430 & 0.92077 \\ \mathrm{H} & -1.04930 & 1.81402 & 0.73392 \\ \mathrm{H} & 1.37690 & -1.60243 & 0.92158 \\ \mathrm{H} & -1.04691 & -1.81542 & 0.73440 \\ \mathrm{H} & -2.07705 & -0.39211 & 0.91990 \\ \mathrm{H} & 2.09489 & 0.00107 & 0.73615 \\ \mathrm{H} & 1.03560 & 1.89415 & -1.40212 \\ \mathrm{H} & -0.70478 & 2.05878 & -1.56766 \\ \mathrm{H} & 1.12462 & -1.84316 & -1.40223 \\ \mathrm{H} & -1.42976 & -1.64047 & -1.56784 \\ \mathrm{H} & -2.15732 & -0.05082 & -1.40343 \\ \mathrm{H} & 2.13664 & -0.41756 & -1.56579 \\ \mathrm{H} & -0.01080 & -1.02938 & 2.78619 \\ \mathrm{H} & -0.88734 & 0.52331 & 2.78653 \\ \mathrm{H} & 0.89530 & 0.50586 & 2.78690 \\ \mathrm{H} & 0.00128 & -0.00008 & -2.74317\end{array}$

Page 18 of 22 


\section{Structure of 18:}

$\begin{array}{lrrc}\mathrm{C} & 0.00000 & 0.00000 & 0.94563 \\ \mathrm{C} & 0.00000 & 0.00000 & -1.72881 \\ \mathrm{C} & -1.44638 & 0.00000 & 0.39604 \\ \mathrm{C} & 0.72319 & 1.25260 & 0.39604 \\ \mathrm{C} & 0.72319 & -1.25260 & 0.39604 \\ \mathrm{C} & -1.42090 & 0.00000 & -1.18646 \\ \mathrm{C} & 0.71045 & 1.23054 & -1.18646 \\ \mathrm{C} & 0.71045 & -1.23054 & -1.18646 \\ \mathrm{C} & 0.00000 & 0.00000 & 2.47811 \\ \mathrm{H} & -1.97669 & 0.88308 & 0.78770 \\ \mathrm{H} & -1.97669 & -0.88308 & 0.78770 \\ \mathrm{H} & 1.75311 & 1.27032 & 0.78770 \\ \mathrm{H} & 1.75311 & -1.27032 & 0.78770 \\ \mathrm{H} & 0.22358 & -2.15340 & 0.78770 \\ \mathrm{H} & 0.22358 & 2.15340 & 0.78770 \\ \mathrm{H} & -1.97195 & 0.88156 & -1.54713 \\ \mathrm{H} & -1.97195 & -0.88156 & -1.54713 \\ \mathrm{H} & 1.74943 & 1.26698 & -1.54713 \\ \mathrm{H} & 1.74943 & -1.26698 & -1.54713 \\ \mathrm{H} & 0.22252 & -2.14854 & -1.54713 \\ \mathrm{H} & 0.22252 & 2.14854 & -1.54713 \\ \mathrm{H} & 1.02502 & 0.00000 & 2.87150 \\ \mathrm{H} & -0.51251 & -0.88769 & 2.87150 \\ \mathrm{H} & -0.51251 & 0.88769 & 2.87150\end{array}$

\section{Structure of 19:}

$\begin{array}{lrrc}\mathrm{N} & 0.00000 & 0.00000 & 1.45793 \\ \mathrm{C} & 0.00000 & 0.00000 & -1.18098 \\ \mathrm{C} & 0.31249 & 1.39588 & 0.92970 \\ \mathrm{C} & -1.36511 & -0.42731 & 0.92970 \\ \mathrm{C} & 1.05262 & -0.96856 & 0.92970 \\ \mathrm{C} & 0.11049 & 1.43888 & -0.59685 \\ \mathrm{C} & -1.30135 & -0.62376 & -0.59685 \\ \mathrm{C} & 1.19087 & -0.81513 & -0.59685 \\ \mathrm{C} & 0.00000 & 0.00000 & 2.94697 \\ \mathrm{C} & 0.00000 & 0.00000 & -2.62903 \\ \mathrm{H} & 1.34378 & 1.59816 & 1.22521 \\ \mathrm{H} & -2.05594 & 0.36467 & 1.22521 \\ \mathrm{H} & 0.71216 & -1.96283 & 1.22521 \\ \mathrm{H} & -0.33923 & 2.08370 & 1.47203 \\ \mathrm{H} & -1.63493 & -1.33563 & 1.47203 \\ \mathrm{H} & 1.97416 & -0.74807 & 1.47203 \\ \mathrm{H} & 0.95055 & 1.94569 & -1.07147 \\ \mathrm{H} & -2.16029 & -0.14964 & -1.07147\end{array}$

Page 19 of 22 


$\begin{array}{rrrr}\mathrm{H} & 1.20974 & -1.79605 & -1.07147 \\ \mathrm{H} & -0.79142 & 1.99509 & -0.85839 \\ \mathrm{H} & -1.33209 & -1.68294 & -0.85839 \\ \mathrm{H} & 2.12351 & -0.31216 & -0.85839 \\ \mathrm{C} & 0.00000 & 0.00000 & -3.87256 \\ \mathrm{H} & -0.13843 & -1.01978 & 3.30490 \\ \mathrm{H} & 0.95237 & 0.39000 & 3.30490 \\ \mathrm{H} & -0.81393 & 0.62977 & 3.30490\end{array}$

\section{Structure of 19-H:}

$\begin{array}{lrrr}\mathrm{N} & -1.50742 & 0.00000 & 0.00000 \\ \mathrm{C} & 1.10431 & -0.00000 & 0.00000 \\ \mathrm{C} & -0.98138 & -0.51923 & 1.33016 \\ \mathrm{C} & -0.98138 & -0.89234 & -1.11474 \\ \mathrm{C} & -0.98138 & 1.41156 & -0.21542 \\ \mathrm{C} & 0.54689 & -0.33130 & 1.40931 \\ \mathrm{C} & 0.54689 & -1.05484 & -0.99157 \\ \mathrm{C} & 0.54689 & 1.38615 & -0.41774 \\ \mathrm{C} & -3.00516 & 0.00000 & 0.00000 \\ \mathrm{C} & 2.56184 & -0.00000 & 0.00000 \\ \mathrm{H} & -1.27602 & -1.56796 & 1.38345 \\ \mathrm{H} & -1.27602 & -0.41412 & -2.04961 \\ \mathrm{H} & -1.27602 & 1.98208 & 0.66617 \\ \mathrm{H} & -1.50939 & 0.02369 & 2.11410 \\ \mathrm{H} & -1.50939 & -1.84271 & -1.03653 \\ \mathrm{H} & -1.50939 & 1.81902 & -1.07756 \\ \mathrm{H} & 1.00979 & -1.24258 & 1.78886 \\ \mathrm{H} & 1.00979 & -0.92791 & -1.97053 \\ \mathrm{H} & 1.00979 & 2.17049 & 0.18168 \\ \mathrm{H} & 0.80390 & 0.47311 & 2.10063 \\ \mathrm{H} & 0.80390 & -2.05576 & -0.64059 \\ \mathrm{H} & 0.80390 & 1.58264 & -1.46004 \\ \mathrm{C} & 3.76288 & -0.00000 & 0.00000 \\ \mathrm{H} & -3.36089 & 0.29141 & -0.98728 \\ \mathrm{H} & -3.36089 & 0.70930 & 0.74601 \\ \mathrm{H} & -3.36089 & -1.00071 & 0.24127 \\ \mathrm{H} & 4.82745 & -0.00000 & 0.00000\end{array}$

\section{Structure of 20:}
C $\quad 0.76819$
$\begin{array}{ll}1.83219 & 0.00244\end{array}$
$\begin{array}{llll}\text { C } & 0.61013 & 0.85239 & 1.19317\end{array}$
$\begin{array}{llll}\text { C } & 0.65617 & 0.83615 & -1.18012\end{array}$
$\begin{array}{llll}\text { C } & -1.39491 & 0.03613 & -0.02731\end{array}$
$\begin{array}{llll}\text { C } & -0.80837 & 0.31011 & 1.21079\end{array}$
$\begin{array}{llll}\text { C } & -0.76066 & 0.29317 & -1.24534\end{array}$
$\begin{array}{llll}\text { C } & 1.46872 & -0.31513 & 0.70956\end{array}$

Page 20 of 22 


$\begin{array}{lrrc}\mathrm{C} & 1.49514 & -0.32434 & -0.64776 \\ \mathrm{H} & -0.01216 & 2.59797 & -0.01793 \\ \mathrm{H} & 1.75734 & 2.30681 & 0.01840 \\ \mathrm{H} & 0.91504 & 1.28124 & 2.15514 \\ \mathrm{H} & 0.99818 & 1.25193 & -2.13531 \\ \mathrm{H} & -2.38159 & -0.43039 & -0.04329 \\ \mathrm{H} & -1.27842 & 0.02513 & 2.14677 \\ \mathrm{H} & -1.19413 & -0.00479 & -2.19486 \\ \mathrm{H} & 1.72495 & -1.15508 & 1.35196 \\ \mathrm{H} & 1.77593 & -1.17286 & -1.26830\end{array}$

\section{Structure of 21:}

$\begin{array}{lrrr}\mathrm{C} & -0.03728 & -0.24546 & -1.66147 \\ \mathrm{C} & -1.22008 & -0.08191 & -1.03841 \\ \mathrm{~N} & 1.17982 & 0.03377 & -1.04308 \\ \mathrm{C} & -1.20127 & 0.32241 & 0.43175 \\ \mathrm{C} & 1.14673 & 0.34126 & 0.40819 \\ \mathrm{C} & -0.02926 & 1.31223 & 0.62801 \\ \mathrm{H} & 0.03516 & -0.58339 & -2.68941 \\ \mathrm{H} & -2.14912 & -0.26630 & -1.56151 \\ \mathrm{H} & 1.97058 & -0.50727 & -1.36380 \\ \mathrm{H} & 2.12128 & 0.73654 & 0.69767 \\ \mathrm{C} & 0.66976 & -0.87885 & 1.18551 \\ \mathrm{C} & -0.67042 & -0.89639 & 1.18388 \\ \mathrm{H} & -1.29878 & -1.71042 & 1.52754 \\ \mathrm{H} & 1.32148 & -1.67646 & 1.52375 \\ \mathrm{H} & -0.00478 & 1.70086 & 1.64794 \\ \mathrm{H} & -0.03649 & 2.13893 & -0.08386 \\ \mathrm{H} & -2.16726 & 0.69146 & 0.77845\end{array}$

\section{Structure of 22:}

$\begin{array}{rrrr}\mathrm{N} & 0.00000 & -0.27180 & 1.58236 \\ \mathrm{C} & -1.20162 & -0.07682 & 1.00513 \\ \mathrm{C} & 1.20162 & -0.07682 & 1.00513 \\ \mathrm{C} & -1.18172 & 0.37256 & -0.43125 \\ \mathrm{C} & 1.18172 & 0.37256 & -0.43125 \\ \mathrm{C} & 0.00000 & 1.34955 & -0.63480 \\ \mathrm{H} & 0.00000 & -0.65579 & 2.51882 \\ \mathrm{H} & -2.08087 & -0.34155 & 1.57063 \\ \mathrm{H} & 2.08087 & -0.34155 & 1.57063 \\ \mathrm{H} & -2.15069 & 0.75200 & -0.75582 \\ \mathrm{H} & 2.15069 & 0.75200 & -0.75582 \\ \mathrm{C} & 0.67566 & -0.86846 & -1.15523 \\ \mathrm{C} & -0.67566 & -0.86846 & -1.15523 \\ \mathrm{H} & -1.31355 & -1.70181 & -1.42676 \\ \mathrm{H} & 1.31355 & -1.70181 & -1.42676\end{array}$

Page 21 of 22 


$\begin{array}{lllr}\mathrm{H} & 0.00000 & 1.73290 & -1.65701 \\ \mathrm{H} & 0.00000 & 2.18360 & 0.07059\end{array}$

\section{Structure of 23:}

$\begin{array}{lrrr}\mathrm{C} & 0.01868 & -0.29913 & 1.65957 \\ \mathrm{C} & -1.20843 & -0.05718 & 1.04383 \\ \mathrm{C} & 1.23765 & -0.10408 & 1.03772 \\ \mathrm{~N} & -1.11912 & 0.33649 & -0.38563 \\ \mathrm{C} & 1.20326 & 0.36374 & -0.40193 \\ \mathrm{C} & 0.02078 & 1.32189 & -0.59707 \\ \mathrm{H} & -0.01181 & -0.67793 & 2.67636 \\ \mathrm{H} & -2.19359 & -0.31292 & 1.39557 \\ \mathrm{H} & 2.17784 & -0.33690 & 1.51708 \\ \mathrm{H} & -2.00354 & 0.71073 & -0.72072 \\ \mathrm{H} & 2.14326 & 0.77753 & -0.76641 \\ \mathrm{C} & 0.69539 & -0.86002 & -1.14006 \\ \mathrm{C} & -0.65316 & -0.86027 & -1.14291 \\ \mathrm{H} & -1.36910 & -1.65683 & -1.28209 \\ \mathrm{H} & 1.31334 & -1.70589 & -1.40682 \\ \mathrm{H} & -0.04977 & 1.70566 & -1.61490 \\ \mathrm{H} & -0.05783 & 2.11134 & 0.14636\end{array}$

\section{Structure of 24:}

$\begin{array}{llll}\text { C } & 0.00000 & -0.28013 & 1.69303\end{array}$

$\begin{array}{llll}\text { C } & -1.22637 & -0.08107 & 1.06174\end{array}$

$\begin{array}{llll}\text { C } & 1.22637 & -0.08107 & 1.06174\end{array}$

$\begin{array}{llll}\text { C } & -1.20046 & 0.28097 & -0.37881\end{array}$

$\begin{array}{llll}\text { C } & 1.20046 & 0.28097 & -0.37881\end{array}$

$\begin{array}{llll}\mathrm{N} & 0.00000 & 1.27977 & -0.58875\end{array}$

$\begin{array}{llll}\mathrm{H} & 0.00000 & -0.56309 & 2.74206\end{array}$

$\mathrm{H} \quad-2.17441 \quad-0.16575 \quad 1.57296$

$\begin{array}{llll}\mathrm{H} & 2.17441 & -0.16575 & 1.57296\end{array}$

$\begin{array}{llll}\mathrm{H} & -2.11469 & 0.74642 & -0.75195\end{array}$

$\begin{array}{llll}\mathrm{H} & 2.11469 & 0.74642 & -0.75195\end{array}$

$\begin{array}{llll}\text { C } & 0.67393 & -0.88102 & -1.18435\end{array}$

$\begin{array}{llll}\text { C } & -0.67393 & -0.88102 & -1.18435\end{array}$

$\mathrm{H} \quad-1.32219 \quad-1.68826 \quad-1.49748$

$\begin{array}{llll}\mathrm{H} & 1.32219 & -1.68826 & -1.49748\end{array}$

$\begin{array}{llll}\mathrm{H} & 0.00000 & 1.66433 & -1.52986\end{array}$

$\begin{array}{llll}\mathrm{H} & 0.00000 & 2.00962 & 0.12072\end{array}$ 